\title{
La migración y el racismo como rasgos complejos de los afrodescendientes del barrio Nigeria
}

\section{Migration and racism as complex features of Afro-descendants in the Nigerian district}

Juan José Rocha Espinoza

Universidad Politécnica Salesiana, Ecuador

Autor para correspondencia: jrocha@ups.edu.ec

Fecha de recepción: 01de Septiembre de 2016-Fecha de aceptación: 20 de Diciembre de 2016

Resumen: El Barrio Nigeria es uno de los sectores que forman la ciudad de Guayaquil, está ubicado en la cooperativa Independencia I-II de la Isla Trinitaria. Este sector está conformado por Afro ecuatorianos que, en su mayoría, son el resultado del fenómeno de la migración dada desde la provincia de Esmeraldas. Como características del barrio se encuentra la pobreza y el desempleo, generados por diferentes factores que han influido a lo largo de la existencia del pueblo afro. En el presente trabajo, se podrá profundizar los rasgos complejos que han surgido en la respectiva investigación de campo, basada en la observación y en las entrevistas que arrojan datos de interés, en los que se evidencia que: la migración, el racismo y otros factores; son el resultado del llamado paradigma de la conquista, marcado por el proceso de occidentalización y globalización. En este sentido los Afro ecuatorianos que optaron por la migración, en su momento se autoconvencieron que, el vivir de las bondades de la tierra y del mar, era vivir en la pobreza; y que el hecho de migrar de su lugar de origen, les ayudaría a salir de la misma. En el caso del Barrio Nigeria la migración contribuyó a que se fortalezcan los círculos de pobreza en la ciudad de Guayaquil, dado que desde sus inicios en los años noventa, hasta hace pocos años atrás, gran cantidad de personas vivían en circunstancias desfavorables referente a localidad y condiciones de vida.

Palabras Claves: Migración; racismo; complejidad; paradigma de la conquista; occidentalización

Abstract: Barrio Nigeria is one of the sectors that make up the city of Guayaquil, is located in Independence I-II cooperative of Trinity Island. This sector is made up of Afro-Ecuadorian that, in most cases, are the result of the phenomenon of migration given from the province of Esmeraldas. As characteristics of the neighborhood is povertyand unemployment, generated by different factors that have influenced throughout the existence of the African people. In this paper, may deepen complex traits that have emerged in the respective field research, based on observation and interviews that cast interesting data, which evidenced that: migration, racism and other factors; They are the result of so-called paradigm of conquest, marked by the process of Westernization and globalization. In this sense Afro Ecuadorian who chose migration, at the time they self-convinced that living the virtues of land and sea, was living in poverty; and the fact that migrate from their home, help them out of it. In the case of Barrio Nigeria migration contributed to the cycles of poverty are strengthened in the city of Guayaquil, given that since its inception in the nineties, until a few years ago, many people living in unfavorable circumstances concerning the quality and living conditions.

Key Words: Migration; racism; complexity; paradigm of conquest; Westernization 


\section{Introducción}

Este trabajo se ha enfocado en el Barrio Nigeria, ubicado en la ciudad de Guayaquil, en lo que se conoce como las Cooperativa Independencia I y II, específicamente en la Isla Trinitaria. Para acceder al mismo, se lo realiza por la vía perimetral. Este sector es considerado como uno en los que se puede evidenciar la pobreza que existe en Guayaquil, dado que Rocha (2014) expone: “(...) el contraste que se puede percibir con el desarrollo central de la urbe y la periferia, puesto que toma menos de veinte minutos desde el centro de la ciudad llegar a esta localidad ubicada en las orillas del Estero Salado" (p.11). Al referirse al contraste, se hace referencia al estado de las viviendas, de las calles, al acceso a servicios básicos, etc.

El contacto que el investigador ha realizado con las personas de este sector se da por medio del Proyecto Salesiano Chicos de la Calle, específicamente, el Padre Marco Paredes, Párroco de la Parroquia Salesiana San Francisco de Sales, que pertenece a la Arquidiócesis de Guayaquil, ha acompañado el proceso de investigación con su experiencia de trabajo con la gente del lugar. Además, este ha sido uno de los actores principales del cambio que el Barrio Nigeria ha experimentado durante estos últimos diez años, como lo evidencia el Diario Expreso en su noticia "El cura que cambió la Nigeria" (Diario Expreso, 2015). En el que expone el trabajo realizado por este en la zona de la Isla Trinitaria, sobre todo con los afros ecuatorianos.

El Barrio Nigeria está conformado en su mayoría por personas afro ecuatorianas, en este sentido el termino afro ecuatoriano quiere decir: “(...) Afro que proviene de África y ecuatorianos, es decir, nacidos en Ecuador" (D’Agostino, 2013: 29). Es importante tener en cuenta que la mayoría de estos decidieron migrar desde la provincia de Esmeraldas, hacia la ciudad de Guayaquil en búsqueda de mejores días. Como dato de relevancia a esta investigación está que la ciudad de Guayaquil, según el Instituto Nacional de Estadísticas y Censos(INEC) con datos del Censo del 2010, posee la mayor cantidad de habitantes que por auto identificación étnica ${ }^{1}$, se consideran afro ecuatorianos, con un total de 256.249,74. Superando inclusive a toda la provincia de Esmeraldas, en la que 234.466,39se autodefinen como Afro ecuatorianos. Además, de la migración, otro rasgo que llama la atención y que está ligado al afro ecuatoriano del Barrio Nigeria, es el racismo, al que están expuestos a pesar de vivir en pleno siglo XXI, esto se podrá detallar más adelante.

El presente trabajo está complementado con el estudio de la complejidad, concepto al que Morin \& Pakman (1994),en su obra Introducción al Pensamiento Complejo, referencian que:"(...) la complejidad es, efectivamente, el tejido de eventos, acciones, interacciones, retroacciones, determinaciones, azares, que constituyen nuestro mundo fenoménico“(p.17).Para comprender mejor el término complejo, este proviene del griego complexus, que quiere decir aquello que se encuentra entre tejido. En la que un acontecimiento o fenómeno que se da esta entre lazado o entretejido con otro u otros. En este sentido el mundo fenomenológico es el Afro ecuatoriano que habita el Barrio Nigeria, que se encuentra relacionado con un tejido de eventos y rasgos, entre los que se destacan la migración y el racismo.

\footnotetext{
${ }^{1}$ Conjunto de rangos en la que el encuestado se ubica según su cultura y costumbres.
} 


\section{Método}

El proceso de investigación, como se muestra en párrafos anteriores, se dio por medio del Sacerdote Salesiano Marco Paredes, quien acompañó el mismo, permitiendo conocer las realidades de las familias del sector. Es importante acotar que este documento no es un primer trabajo académico realizado por el autor en el Barrio Nigeria, ya existe caminar investigativo y un convivir con la comunidad, llevando a utilizar la metodología analítica sintética, complementada con la técnica de la observación fenomenográfica, que Morín (1995) expone:

"La observación debe llevarse a cabo tanto sobre los centros de la vida social como sobre los hogares individuales y debe acompañar a otros empeños indagatorios, aun manteniéndose autónoma. Debe tender, idealmente, a cubrir la totalidad del fenómeno estudiado, incluido el observador en su observación" (Morín, 1995, p.194).

Además, de la observación fenomenográfica, se aplicó la técnica de la entrevista en la que se pudo determinar información muy valiosa de parte de los habitantes del sector, esta entrevista fue estructurada en diferentes ámbitos, pues los datos también serán utilizados para una tesina de maestría. El método aplicado es netamente cualitativo, por el hecho de ser cualitativo, es analítico complementado con el tipo de estudio descriptivo, permitiendo una mejor asimilación de los datos obtenidos.

\section{Resultados}

El presente apartado se podrá conocer los resultados alcanzados en el proceso de interacción con la comunidad. Teniendo como premisas a la migración y el racismo como rasgos complejos de quienes habitan el Barrio Nigeria.

\section{Migración}

Dentro de la historia del Barrio Nigeria se encuentra el fenómeno de la migración, es importante puntualizar que en gran porcentaje las personas que habitan este sector son Afro ecuatorianas que, en su mayoría, son el producto del desplazamiento migratorio desde la provincia de Esmeraldas (específicamente de los siguientes sectores: Borbón, Limones, Collón Eloy, San Lorenzo,Timbiré, Santoya, Selva alegre, Maldonado, San José de Tagua),hacia la ciudad de Guayaquil. Llegando a ubicarse en su conjunto en lo que se conoce como la vía perimetral, en este sentido expone Villavicencio (2012) que tras la construcción de la misma ésta “(...) incentivó a nuevas invasiones siguiendo el trazado de la misma, proceso que se ha mantenido durante las dos últimas décadas. En el sur-oeste de la urbe hubo la ocupación de la Isla Trinitaria" (Villavicencio, 2012, p.111). Villavicencio (2012) solo hace una descripción de la situación que se da tras la construcción de la vía. Sin embargo, el haberse adentrado en la comunidad permite determinar que cuando estas personas empezaron a poblar el sector se ubicaron a la orilla del Estero Salado, sobre todo encima del mismo, en casas de caña.

Esta migración trajo consigo el fortalecimiento de los círculos de pobreza con los que cuenta la ciudad de Guayaquil, pues hace más de diez años era baja la calidad de vida de estas 
personas, como se corrobora en el 2004 cuando un diario de la ciudad, EL UNIVERSO, saca como noticia: "Nigeria", y el hambre en los rostros (Santana, 2004).Siguiendo el tema de la calidad de vida de quienes habitan el sector, una de las personas que fue entrevistada comenta como vivían en esos tiempos: “(...) aquí las cosas eran agua, puente, y vivíamos encima del estero. La marea bajaba y subía no teníamos agua, no teníamos luz, nos ingeniábamos para poner la luz a expensas de la marea.(P. Prado, comunicación personal, 13 de febrero del 2016). De esta manera se evidencia el escenario en el que vivan estas personas. Además, se puede ver como la realidad compleja emitida por el rasgo de la migración se entrelaza con la pobreza a la cual se expusieron.

Las causas de la migración que aducen en el momento en que se les entrevista es constante, pues estas son: situación económica difícil y falta de oportunidades laborales. En este sentido, responden a la pregunta ¿Por qué decidieron salir de su lugar de origen? Como en el caso de Bertha Valencia, expresa que: “(...) la economía estaba dura, entonces me vine para ver si hay mejores días" (B. Valencia, comunicación personal, 22 de marzo del 2016).Otro morador del sector expresa "(...) no teníamos las oportunidades en la cuidad de donde salí" (J. Castillo, comunicación personal, 25 de marzo del 2016), haciendo referencia a Collón Eloy, lugar donde proviene el mismo. También un joven que migró desde su infancia con su familia haca la ciudad de Guayaquil expresa que la gente opto por “(...) buscar una estabilidad económica en los sectores de esmeraldas como Borbón, Timbiré, Santoya. La gente vive del cacao, del verde y de sacar madera (...) por eso la gente prefiere venir a trabajar de obrero por una estabilidad”. (R. Angulo, comunicación personal, 13 de febrero del 2016).

Es comprensible el hecho de la migración de estas personas, puesto que según cifras de la Encuesta de Condiciones de Vida (ECV) 2006, citadas por la Defensoría del Pueblo (2012)indican que “(...)el 48.7\% de los afro ecuatorianos viven por debajo de la línea de pobreza por consumo, es decir, 5 de cada 10 hogares afros apenas poseen $\$ 56$ dólares mensuales para su consumo básico"(Defensoría del Pueblo, 2012, p.65). Bajo estos indicadores se logra conocer la realidad del pueblo afro a nivel nacional, el cual ha influido para que se dé la migración.

Dentro de la investigación surgen factores interesantes, dado que entrevistados consideran que en sus lugares de origen no se haya vivido en la pobreza absoluta, debido a que vivían de las bondades de la tierra y del mar. Se dedicaban al cultivo de sembríos y a la pesca como expresa uno de los entrevistados: "no había pobreza, sino que hay gente que quería otro estilo de vida porque allá se vivía de la pesca. Y la gente busco otras oportunidades". (P. Prado, comunicación personal, 13 de febrero del 2016).Además, expone otra persona que: "el trabajo en el campo es muy cansado, para sobrevivir se necesita tener cultivos y para vender, se tenía que madrugar para poder salir en canoa a vender verde o cualquier cultivo". (Y. Angulo, comunicación personal, 22 de marzo del 2016).

Con lo antes mencionado se puede considerar que comienza a emerger la herencia occidental $^{2}$,respondiendo al paradigma de la occidentalización, en el que estas personas se auto-

\footnotetext{
2 “(...) teoría de la occidentalización que afirma, en síntesis, que en el proceso de aculturación lo indígena fue suprimido por la imposición de normas cristianas que transformaron tanto las formas de sujeción como los esquemas de vida y de conocimiento”.(Terán, 2007)
} 
convencieron de vivir en la pobreza, pues el paradigma les obliga, por ende deciden salir de sus hogares y dejar de trabajar la tierra.

2“(...) teoría de la occidentalización que afirma, en síntesis, que en el proceso de aculturación lo indígena fue suprimido por la imposición de normas cristianas que transformaron tanto las formas de sujeción como los esquemas de vida y de conocimiento". (Terán, 2007)

\section{Racismo}

El racismo es uno de los rasgos complejos que se han encontrado en los afro ecuatorianos que habitan el Barrio Nigeria, sobre todo expresan haber sido víctimas de este tipo de actos discriminatorio. E inclusive argumentan algunos de ellos no encontrar trabajo por esta situación, lógicamente no justifica el desempleo en común que acosa a estas personas, sino es un sentir basado en experiencias de los entrevistados. Es así como uno de ellos expresa: “(...) no nos dan trabajo porque somos negros y piensan que le vamos a robar" (D. Caicedo, comunicación personal, 22 de marzo del 2016).También Patricia cuando se le pregunta sobre la misma situación indica: “(...) piensan que los afros somos ladrones, no nos conocen” (P. Prado, comunicación personal, 13 de febrero del 2016).

No obstante, no solo alegan haber sido discriminadas racialmente por personas nativas de la ciudad de Guayaquil, sino hay caso de personas extranjeras que han expresado su rechazo como es el caso de Denisse, que expone: “(...) fuimos a un restaurante de comida china y había vacante de dos personas y el chinito salió a decir que no queremos gente del color de su piel" (D. Caicedo, comunicación personal, 13 de febrero del 2016). Sin duda alguna esta situación del racismo es profunda, a pesar de que la ciudad de Guayaquil es considerada una ciudad moderna. Sin embargo, existe este tipo de situaciones en las que se debe tomar conciencia sobre el actuar personal y comunitario.

Basado en una observación de campo a un grupo numeroso de jóvenes compuesto por personas afrodescendiente y mestizas que rodean los 15 a 18 años, se pudo analizar una asociación entre jóvenes afro, excluyéndose de toda relación con los mestizos; a diferencia de las demás jóvenes "no afro", quienes si buscaban interactuar sin prejuicios. Esta resistencia de asociarse entre afros se podría considerar como resultado del racismo que se ha dado durante generaciones.

Sobre el racismo y las construcciones sociales del mismo Whitten (1999), expone: "(...) cuando esta distinción introduce en nuestro discurso conceptos tales como 'blanco', 'indio', 'negro', o paradójicamente, 'mestizo', invocamos y evocamos inconscientemente el fuerte paradigma mental de la Conquista". (Whitten, 1999, p.48)Es así que se puede determinar que se responde al paradigma de la Conquista, dado que está determinado por la herencia obtenida basada en una mayoría denominada como blanca.

\section{Conclusiones}

Se determina que la migración y el racismo son rasgos complejos pues son fenómenos entretejidos al afro ecuatoriano del Barrio Nigeria. La migración justificada por 
la situación económica (pobreza) y la falta de oportunidades, simplemente responden a la teoría de la occidentalización. Puesto que deciden cambiar sus esquemas de vida y responder a la occidentalización, teniendo en cuenta que el vivir de la tierra y del mar no significa vivir en pobreza. El racismo ha estado arraigado por generaciones en la sociedad ecuatoriana y guayaquileña. Lamentablemente se responde a un paradigma de la conquista, en la que se determinó los diferentes calificativos étnicos, sin embargo, se debe y se tiene que romper aquel paradigma.

\section{Bibliografía}

D’Agostino, A. (2013). Identidad, cultura y cimarronaje en las insurgencias/emergencias del pueblo afroecuatoriano 1980-2011.

Diario Expreso, (2015). El cura que cambió la Nigeria. Recuperado a partir de http://expreso.ec/historico/el-cura-que-cambio-la-nigeria-NRGR_8131638.

Defensoría del Pueblo. (2012). El pueblo afrodescendiente en el Ecuador. Quito: DPE. [En línea]. Disponible en: http: //repositorio.dpe.gob.ec/handle/39000/64.

Morin, E., \& Tortella, J. (1995). Sociología. Barcelona: Tecnos.

Martínez Terán, T. (2007). El imaginario del colonizador. Argumentos (México, DF), 20(53), 143-167.

Rocha, J. J. (2014). Barrio Nigeria: De la caña al cemento y de la miseria al microemprendimiento.

Santana, F. (2004). "Nigeria”, y el hambre en los rostros. El Universo, P1.

Villavicencio, G. (2012). Las invasiones de tierras en Guayaquil: historia y coyuntura política (Política pública).

Whitten Jr, N. (1999). Los paradigmas mentales de la conquista y el nacionalismo: la formación de los conceptos de las 'razas' y las transformaciones del racismo. Ecuador racista, 45-72. 